\title{
Philosophiques
}

\section{" Et la religion le remplit de fureur... " : Les déterminations idéologiques, polémiques et politiques, du Mahomet de Voltaire.}

\author{
Josiane Boulad-Ayoub
}

Volume 17, numéro 2, automne 1990

URI : https://id.erudit.org/iderudit/027119ar

DOI : https://doi.org/10.7202/027119ar

Aller au sommaire du numéro

Éditeur(s)

Société de philosophie du Québec

ISSN

0316-2923 (imprimé)

1492-1391 (numérique)

Découvrir la revue

Citer cet article

Boulad-Ayoub, J. (1990). « Et la religion le remplit de fureur... » : Les déterminations idéologiques, polémiques et politiques, du Mahomet de Voltaire. Philosophiques, 17(2), 3-22. https://doi.org/10.7202/027119ar
Résumé de l'article

Il s'agira, en examinant sa tragédie de 1742 avec laquelle débute l'intérêt explicite de Voltaire envers le Prophète de l'Islam, de retracer ce qu'avec Mahomet ou le fanatisme, Voltaire, un Voltaire critique d'abord de sa société et futur auteur du Traité sur la Tolérance, met véritablement en scène, ou plus précisément, en arrière-scène pour les besoins idéologiques de sa dénonciation. On commentera, chemin faisant, les aspects polémiques de ces attaques contre la superstition et le fanatisme religieux ainsi que les conséquences politiques qu'elles ont eues sur son propre contexte historique pour finir par poser, en conclusion, la question de leur actualité, devant la résurgence contemporaine des intégrismes quels qu'ils soient, et de la mort et de la violence qui les accompagnent inéluctablement. 
PHILOSOPHIQUES, Vol. XVII, Numéro 2, Automne 1990

\title{
ARTICLES \\ «ET LA RELIGION LE REMPLIT DE FUREUR...» LES DÉTERMINATIONS IDÉOLOGIQUES, POLÉMIQUES ET POLITIQUES, DU MAHOMET DE VOLTAIRE.
}

\author{
par Josiane Boulad-Ayoub
}

\begin{abstract}
RÉSUMÉ. Il s'agira, en examinant sa tragédie de 1742 avec laquelle débute l'intérêt explicite de Voltaire envers le Prophète de l'Islam, de retracer ce qu'avec Mabomet ou le fanatisme, Voltaire, un Voltaire critique d'abord de sa société et futur auteur du Traité sur la Tolérance, met véritablement en scène, ou plus précisément, en arrière-scène pour les besoins idéologiques de sa dénonciation. On commentera, chemin faisant, les aspects polémiques de ces attaques contre la superstition et le fanatisme religieux ainsi que les conséquences politiques qu'elles ont eues sur son propre contexte historique pour finir par poser, en conclusion, la question de leur actualité, devant la résurgence contemporaine des intégrismes quels qu'ils soient, et de la mort et de la violence qui les accompagnent inéluctablement.
\end{abstract}

ABSTRACT. I intend to bring out the ideological determinations of Voltaire's Mabomet ou le fanatisme and of his attacks on religion. I will analyze his polemical stand against fanaticism and superstition in respect to the historical and societal context as well as the political consequences of this denunciation by the future author of the Traité sur la Tolérance. In conclusion the problem of the actuality of the Voltairian struggle will be examined in respect to the contemporary resurgences of religious fundamentalist views.

* Le texte qui suit a été rédigé en vue de la participation de l'auteur à un colloque international organisé au Caire, en 1990, par le Centre d'études françaises de cette ville, sur le thème: La réception de Voltaire et de Rousseau en Égypte. 


\section{«...Mahomet [...] tel qu'il a plu aux philosophes, et tel qu'il a révolté les dévots ${ }^{1}$ "}

Dans l'ceuvre de Voltaire, l'Histoire de Charles XII puis Zaïre marquent les débuts de ses rencontres avec l'Islam en même temps que chacun de ces textes préfigurent les pôles interactifs de son interprétation du monde musulman : objectivité de l'historien des idées mâtinée des impératifs de la lutte idéologique du philosopbe, points de vue a priori contradictoires mais que son pragmatisme parvient dynamiquement à concilier. Et que ce soit devant le problème de savoir ce que c'est d'être musulman, Turc, Persan, Arabe ou devant d'autres questions de philosophie religieuse et morale aux conséquences politiques tout aussi lourdes, Voltaire se montre, comme partout dans ses écrits, le plus éclatant représentant de l'activité militante de la Raison tel que l'entend et la pratique le siècle de la Critique, quand esprit philosophique rimait avec science et progrès des Lumières pour affranchir l'homme de toutes les espèces de tyrannie. L'Islam, ses personnages, ses valeurs, ses mœurs, vont donc, selon les circonstances de l'histoire intellectuelle et politique de son époque et de ses propres partis-pris, servir à Voltaire tantôt d'objet de connaissance et d'approfondissement scientifique, tantôt comme un arsenal d'exemples et de figures où puiser pour alimenter la guerre contre l'infâme.

Autrement dit, il convient, pour l'explication, de suivre ces deux grandes lignes de force complémentaires qui déterminent symboliquement la réception, si j'ose dire, de l'Orient par Voltaire, et en particulier de dégager, là où elles semblent converger de la façon la plus révélatrice quand Voltaire étudie la religion dominante dans l'empire ottoman, l'Islam, les aspects contrastés qu'il donne de son fondateur. De Mabomet au dernier jugement de Voltaire sur l'Islam dans sa correspondance de $\mathbf{1 7 7 2}$, en passant par l'Essai sur les mours, l'article "Alcoran" de son Dictionnaire, ou Candide, le plus "turc" de ses Contes, on peut suivre les relations complexes de Voltaire avec la religion musulmane et le Prophète ; elles ne laissent pas d'avoir pour

1. Ce sous-titre de meme que les deux autres sous-titres du texte de l'article sont des citations tirées des lettres de Voltaire à Frédéric Il et au comte d'Argenson au sujet de sa tragédie Mabomet. In Voltaire, Correspondance, édition Théodore Besterman, Bibliotheque de la Pleiade, NRF-Gallimard, tome II (17391748), Paris, 1977. Il s'agit, dans I'ordre de leur apparition, des lettres no 1617 , à Frédéric II, 29 août 1742, n० 1614, a D'Argenson, 22 aout 1742, no 1474, à Frédéric II, 20 janvier 1740. 
arrière-fond sa critique de la société et de la culture de la France du XVIII ${ }^{e}$ siècle. Une constante cependant : si, lorsqu'il regarde les événements de son œil historique et qu'il polémique contre le christianisme ou les mœurs chrétiennes, Voltaire tire argument des dogmes et des mours du monde musulman pour renvoyer au type de déisme qu'il défend ou à la religion et la morale naturelles qu'il prône, en revanche le personnage de Mahomet restera pour Voltaire à chaque fois qu'il en parle, «un sublime et hardi charlatan *. Ici, l'objectivité historique le cédera toujours à son obsession du fanatisme, à son acharnement à dénoncer le prêtre sanguinaire et superstitieux qui abuse de la crédulité du peuple et le conduit à toutes sortes de crimes. Ici c'est le Voltaire ardent apôtre de la tolérance et «ami du genre humain qui prend le pas et ne traite du Mahomet historique que comme prétexte commode pour peindre, sous son masque, les ravages que commet dans l'Histoire, le fanatique ambitieux. Il le dira lui-même en 1776 : «J'ai fait Mahomet beaucoup plus méchant qu'il n'était"

Mais précisément qu'est-ce qui a conduit Voltaire à noircir intentionnellement la personne-clé d'une religion dont il chante par ailleurs les louanges, dont il admire la définition de Dieu et la sagesse des préceptes qu'elle contient? En un mot, s'il fait de Mahomet un monstre, de quelles répulsions l'a-t-il donc chargé? Nous nous proposons d'esquisser ici une réponse à cette délicate question; l'examen ne voudra cependant pas déborder les aspects strictement politiques du problème en cause. En effet, étant donné les limites imposées par le cadre d'un bref exposé, nous nous pencherons sur une des dimensions seulement que subsume la conception voltairienne de Mahomet pour faire l'analyse de ses déterminations idéologiques. C'est dire que nous ne nous attacherons pas ici à comparer, du point de vue cognitif, ce que la science et l'histoire nous disent de Mahomet ou de la religion musulmane avec le discours de Voltaire; de même, nous ne chercherons pas non plus à approfondir les thèses de sa philosophie religieuse, chrétienne ou mahométane. Il s'agira plutôt, en examinant sa tragédie de 1742 avec laquelle débute l'intérêt explicite de Voltaire envers le Prophète de l'Islam, de retracer ce qu'avec Mabomet ou le fanatisme, car tel est le titre complet de la pièce, Voltaire, un Voltaire critique d'abord de sa société et futur auteur du Traité sur la Tolérance, met véritablement en scène, ou plus précisément, en arrière-scène pour les besoins de sa dénonciation. On commentera, chemin faisant, les aspects polémiques de ces attaques contre la super- 
stition et le fanatisme religieux ainsi que les conséquences politiques qu'elles ont eues sur son propre contexte historique pour finir par poser, en conclusion, la question de leur actualité, ou même, à proprement parler, de leur éternité devant la résurgence contemporaine des intégrismes quels qu'ils soient, et de la mort et de la violence qui les accompagnent inéluctablement, aujourd'hui au temps des ayatollabs comme hier au temps de la Ligue dont les horreurs faisaient encore trembler à l'approche de chaque 24 août ${ }^{2}$, l'auteur de la Henriade.

\section{"Les Turcs de Paris..."}

On sait la carrière singulière des représentations de Mabomet, le succès éclatant à Lille où la tragédie fut d'abord jouée, le tollé à Paris, les réactions contradictoires des spectateurs ; ce tumulte des interprétations ${ }^{3}$ signifiait assez par lui-même que l'œuvre se jouait sur plusieurs registres et que les sentences nerveuses de Mabomet disaient passionnément leur fait à des puissances redoutables mais reconnaissables. Les « gens, nous rapporte Desnoiresterres, trouvèrent là des traits hardis contre la religion, le gouvernement et la morale établie ${ }^{4}$.» Les uns, les dévots naifs qui ne comprenaient pas très clairement les intentions combattantes de Voltaire et sa manière de conduire la révolution des esprits, ou bien encore les complices gagnés aux idées "philosophiques* qu'il mettait de l'avant sous le masque de Mabomet, applaudissaient et entraient dans le jeus, les

2. RenÉ POMERLEAU souligne * le sens maladif * que Voltaire avaic du fanatisme. I nous rappelle, dans son Voltaire par lui-même, Le Seuil, Paris, 1955, p. 39, qu' «à Ferney, il [Voltaire] avait la fièvre, tous les ans, à l'anniversaire de la Saint-Barthélémy. Il l'a dit lui-même, plusieurs fois, et un témoin l'a confirmé à l'approche du 26 aoot, Voltaire tombait dans la prostration et s'alitait *.

3. La pièce de Voltaire provoqua aussi, de la part de ses contemporains un déluge d'écrits pour la combattre ou, au contraire, la défendre: Villaret, Lettre à $M$. de Voltaire sur sa tragédie de Mabomet, 1742; Abbé Cahagne, Lettre d'un comédien de Lille sur la tragédie de Mahomet, 1742; Sentiments d'un spectateur sur la piece de Mabomet, 1742 (in Amusements du coeur et de l'esprit, t. XIII); Lettre écrite a M. le comte de *** au sujet de la tragédie de Mabomet, 1742

4. G. DESNOIRESTERRES, Voltaire et la societé au XVIII siècle, tome II, Voltaire d Cirey, Slatkine Reprints, Genève, 1967, pp. 339-40.

5. Dans les amusements du coeur et de l'esprit, t. XIV, 1742, p. 427, l'auteur anonyme d'une Lettre écrite d $M$. le Comte de..., nous dit qu'après la suspension des representations de Mabomet a Paris, on fit jouer Polyeucte à la place, mais que les spectateurs n'applaudirent dans toute la tragédie de Corneille que les imprécations de Stratonice contre les Chrétiens et la déclaration tolérante de Sévère. 
autres, méfiants à l'avance du philosophe ${ }^{6}$, s'ébranlèrent, s'indignèrent, et, passant à l'attaque, réussirent, malgré les efforts des amis de Voltaire, et en particulier de Madame du Châtelet, à faire arrêter les représentations ${ }^{7}$. Voltaire retourne alors à Cirey, écrivant à toute l'Europe éclairée pour faire part de cette nouvelle aventure du Tartuffe et accuser ses adversaires : les hypocrites et les réactionnaires, ennemis de la vérité et de la liberté de penser. Les Turcs de Paris, comme il les appelle, ont fait interdire sa pièce. Il a été, dit-il, victime des jansénistes et des convulsionnaires en robe longue. Aussi, en guise de contre-attaque, il va, décide-t-il, "dédier Mabomet au pape" et se faire "évêque in partibus infide. lium attendu que c'est là mon véritable diocèse * 8 .

Voltaire tenait beaucoup à son Mabomet, à la dissimulation habile de sa critique, au tour substantiel et profond qu'à son gré, il avait réussi à imprimer à son sujet; ce qui lui semblait conférer à la pièce un caractère de grandeur politique tout à fait dans le goût cornélien, le modèle de Voltaire dans l'art dramatique et qu'il essaie d'égaler, voire de dépasser. Il était convaincu qu'il avait là un chef d'œuvre et écrivait à d'Argental, en juillet 1740 : «Je commence à entrevoir que Mabomet sera sans aucune comparaison ce que $j$ 'aurai fait de mieux... ${ }^{*}$. Pour des raisons littéraires et

6. L'abbé Le Blanc, un an avant que Voltaire ne demande l'autorisation du cardinal de Fleury, mandait à Bouhier : Il est a présent question du Mabomet de $M$. de Voltaire, M. de Marville n'en veut pas prendre l'approbation sur son compte, et il est à présent entre les mains de $M$. le Cardinal; le prophète est confondu dans la pièce, mais il est vrai qu'il faut regarder de près à ce que fait le compère Arouet. latet anguis in berba... Rapporté par G. Desnoiresterres, Voltaire et la société au XVII'e siecle, op. cit., p. 336.

7. Ce sera Voltaire lui-même, précisons-le, qui finira par retirer sa tragédie, cédant par-là aux pressions exercees contre lui. M. de Marville, en effet, le lieutenant de police, suivant en cela l'habile conseil du ministre Maurepas qu'il avait consulté sur l'affaire, avait communiqué à Voltaire la lettre du procureur général du Parlement de Paris au sujet de son Mabomet, aux *épithètes fácheuses ", et lui avait fait part des allusions de Joly de Fleury, le procureur en question, à un arret éventuel du Parlement pour décréter l'exécution des Lettres Philosophiques, parues en 1734. Même si Voltaire s'était ainsi incliné, le scandale provoqué par Mabomet contribua à l'échec de son auteur à l'Académie en $\mathbf{1 7 4 3}$. Il reste qu'il faut signaler que la pièce fut reprise, huit ans plus tard, en 1751, mais, cette fois, sans que personne ne songeal à l'interdire. On y verra une autre preuve de la rapidité avec laquelle les idées philosophiques se propageaient alors.

8. VOLTAIRE, Correspondance, op. cit., lettre no 1614, à d'Argental, 22 aout 1742 Voltaire aura l'irrévérence de réaliser sa plaisanterie et se permettra de dédier sa pièce * victime des jansénistes * à Benoît XV; celui-ci accepta la dédicace et lui enverra même, en 1745, des "médaillons sacrés*. Voltaire s'était permis, en attendant, de faire circuler et d'imprimer à Paris pendant la querelle du Mabomet, une fausse approbation pontificale de sa pièce!

9. VOLTAIRE, Correspondance, op. cit., lettre no 1408, au Comte d'Argental, 12 juillet 1740 . 
artistiques ${ }^{10}$ d'abord, mais, tout autant, la forme servant le fond, parce qu'il lui semblait que les thèmes qu'il y développait - « j'ai voulu faire voir par cet ouvrage à quels horribles excès le fanatisme peut entraîner des âmes faibles conduites par un fourbe ${ }^{11}$ n - servaient, de manière forte et utile, * la vérité et le genre humain ${ }^{12}$ \%.

Sa correspondance pendant les années qui précèdent les représentations à Lille et qui font mention de l'œuvre qu'il a en chantier, témoigne du soin extrême avec lequel il travaille sa tragédie, la révisant et la corrigeant sans cesse, donnant "plusieurs leçons", les envoyant à des correspondants choisis, surtout au comte d'Argental, pour leur demander conseil, bref, ne négligeant jamais sa peine et toujours prêt, même après le succès lillois, à donner à sa pièce "encore quelques coups de lime", comme il dit ${ }^{13}$.

10. Voltaire est très conscient de la nouveauté du sujet et de l'action traités. Emprunter son canevas a l'histoire orientale lui permettait, en plus de sacrifier aux turqueries au gout du jour, de s'éloigner surtout avec le " gros vin de Mabonet* (Voltaire, Correspondance, op. cit. , lettre no 1334) de l'imitation sempiternelle sur la scène dramatique des héros de l'antiquité grecque et romaine. Il reviendra a plusieurs reprises sur la nouveaute qui distingue la pièce. Ainsi il écrit à ses amis d'Argental (Correspondance, op. cit., lettre no 1337) en février 1740 , qu'il *y a plus [par comparaison avec son autre pièce turque Zulime] de neuf, plus d'invention, plus de choses, dans une seule scène de ce drole-la que dans toutes les lamentarions amoureuses etc.....; ou bien encore à d'Argental, le 12 juin 1740 (Correspondance, op. cit., lettre no 1381), il lui avoue que je n'ai jamais mis mes complajsances que dans Mabomet. J'aime les choses d'une espèce toute neuve. . Une autre caractéristique de sa tragédie que met en relief Voltaire est son côté shakespearien, si l'on peut dire, côté qui ne laisse pas d'ailleurs de préfigurer le romantisme et la propension des auteurs du XIXe siècle à s'enchanter du *sublime * qui signale l'horreur magnifique. Une lettre très curieuse, à cet égard, de Voltaire à Ferriol (Correspondance, op. cit., lettre no 1407), en juillet 1740 , compare certaines scènes de son Mabomet avec la manière de Rembrandt: "Cependant le moment où Mahomet se justifie aux yeux du peuple par ce faux miracle de la mort de Séide, et cet art étonnant de conserver sa réputation par un crime, est à mon gré une si belle horreur, que je vais tout sacrifier pour peindre ce sujet de Rimbran [sic. Voltaire écrit phonétiquement le nom du peintre selon la prononciation du temps] de ses couleurs véritables $n$.

11. VOLTAIRE, Correspondance, op. cit., lettre no 1618 , à César de Missy, 1 septembre 1742 .

12. Ibidem.

13. J'ai compré quelque 28 lettres échelonnées au long des années 1739 et 1740 , et 20 lertres pour l'année 1741 . Après le succès de Lille en mai 41 , Voltaire reprendra encore la tragédie pour la corriger avant sa représentation a Paris. Il s'agit des no 1208 (la première lettre où Voltaire fait allusion à la nouvelle pièce qu'il entreprend est de mars 1739), 1272, 1276, 1282, 1290, 1299, 1315, 1318 (la première lettre de l'année 1740), 1327, 1328, 1332, 1334, 1335, 1336, 1337, $1338,1342,1343,1344,1345,1354,1358,1359,1364$ (qui signale la première version complète de son ve acte), 1371, 1373, 1381, 1394, 1400, 1407, 1408, 1474 (adresséc à Frédéric II et qui deviendra la préface de Mabomet). Les 20 lettres de l'année 1741, pour leur part, portent les numéros 1477, 1478, 1486 , $1489,1491,1499,1509,1517,1518,1519,1521,1528,1529,1531,1532,1533$, $1538,1541,1552,1556,1563,1580$. In Correspondance, opus cit. Toutes ces lettres font état plus ou moins longuement des préoccupations diverses que lui 
Aussi bien cette correspondance qui accompagne la préparation de son Mabomet fait-elle apparaître, à travers les remarques sur les caractéristiques plus formelles de la pièce, les stratégies que Voltaire met au point pour faire sauter les barrières que les Welches opposent à la marche de la Raison ${ }^{14}$. De même, les commentaires de Voltaire à ses correspondants, et, en particulier, sa lettre de janvier 1740 à Frédéric II qui deviendra la préface du Mabomet imprimé, révèlent sans détours beaucoup de ses intentions sur le plan des cibles politiques visées. Si leur réalisation plut aux philosophes et révolta les dévots ${ }^{15}$, la querelle du nouveau Tartuffe que déclenche l'apparition de sa tragédie, et les réactions de Voltaire contre ses adversaires, sont à leur tour enregistrées, sur le vif, pourrait-on dire, par la correspondance immédiatement postérieure aux représentations de Paris, dont par les lettres de l'année 1742 auxquelles il faut ajouter les échanges avec le Vatican en $1745^{16}$. La

cause Maboniet et les commentaires de Voltaire nous renseignent autant sur les cibles politiques qu'il visait expressément avec sa tragédie que sur la conception qu'il se faisait des ressorts de l'art dramatique de meme que sur le govt de l'époque. On y retrouve les deux facettes inséparables de son génie : l'íntellectuel engagé, pour le dire en termes contemporains, et l'écrivain incomparable en même temps qu'acharné au travail, nous livrant au fil des réflexions sur l'élaboration de son ceuvre une sorte de Poétique vivante et informelle.

14. Voltaire sait que la critique intellectuelle, quelque profonde et vraie soit-elle, ne suffira pas d'elle-meme a lui gagner le public. Les pbilosopbes sont a un contre cent dans une salle de spectacles ... pour remuer le cour des bommes, il faut de l'amour et de la religion patbétique, écrit-il, après l'échec d'Érypbile. Voltaire désire plaire pour convaincre, et le grand ressort est d'émouvoir, meme lorsque cela ne serait pas a l'bonneur de la raison et que la circonspection l'oblige a ne pas dire tout ce qu'on pourrait dire. (Correspondance, opus cit., lettre à d'Argenson, no 1327, 26 janvier 1740). Il faut également tâcher de dépayser le public (Correspondance, opus cit., lettre a Mademoiselle Quinault, no 1364 , le 19 avril 1740) pour faire passer les vérités de Mabomet, confie Voltaire à sa comédienne principale, et ajoute que Mabomet n'est pas le róle intéressant. Ce serait plutot le personnage de Séide qui l'assumerait, et le thème central de la tragédie, celui de la suggestion criminelle. La pièce, vue sous ce jour, détaillerait les ressorts de la séduction d'un jeune aveugle au grand cour par un chef de parti pour en faire un assassin. Mabomet est dirigé avant tout contre " les maximes infernales qui ont mis le couteau à la main des Poltrot, des Ravaillac, des Chatel... De meme dans sa lettre à Frédéric II de janvier 1740, déjà citée, Voltaire développe l'idée que Mabomet ne fait que reprendre mais sous d'autres noms l'épisode de Jacques Clement. Il cite alors de nombreux exemples d'assassinats inspirés par le fanatisme et les sectateurs d'un Dieu vengeur.

15. VOLTAIRE, Correspondance, op. cit., lettre n० 1617 , à Frédéric II, 29 aout 1742. Voltaire annonce au roi de Prusse la transcription de son Mabomet: *Je vais pendant les trois ou quatre jours que je [sic] condamné a rester dans mon lit faire transcrire le Mabomet tel qu'il a été joue, tel qu'il a plu aux philosophes, et tel qu'il a révolté les dévots. * Et Voltaire, se comparant à Molière et le sort de sa pièce à * l'aventure de Tartuffe *, continue en désignant ses persécuteurs: * les fanatiques se sont soulevés contre moi *

16. Revenons dans Voltaire, Correspondance, op. cit., les lettres no 1585,1614 (du 22 août 1742, à d'Argental, où Voltaire annonce ses intentions de s'établir à Paris contre les jansénistes er les convulsionnaires, évëque in partibus infidelium; notons qu'à partir de cette date, Voltaire va faire débuter ironiquement 
cinquantaine de lettres que Voltaire envoie à ses correspondants, amis, comédiens, gens de lettres, protecteurs..., pendant les années 1739 à 1741, nous renseignent entre autres, sur les enjeux idéologiques de Mabomet, alors que les quelques quinze lettres datées de 1742 précisent encore mieux les forces en présence ainsi que l'impact contemporain qu'eurent, en attendant le retentissement encore plus grand de l'affaire Calas, la condamnation par Voltaire des horreurs du fanatisme religieux et sa lutte active pour l'avènement de la tolérance et de la liberté dans une société débarrassée de la tyrannie conjointe $d u$ trône et de l'autel.

En commentant quelques-unes des lettres qui viennent d'être mentionnées et en les conférant avec les articles du Dictionnaire portatif qui développeront plus tard les mêmes thèmes de concert avec la lutte de l'Encyclopédie, du moins sur le sujet du fanatisme, je voudrais maintenant cerner de plus près l'action de Voltaire contre l'imposture et la persécution tout en soulignant ses aspects les plus significatifs par rapport à l'époque. À quelle sorte de religion, de gouvernement et de morale s'en prend donc le philosophe, lorsqu'il nous dépeint, sous le nom de Mabomet, la triste histoire de Séide, parricide sans le savoir, âme innocente corrompue par les intrigues politiques d'un faux prophète?

Les premiers contacts de Voltaire avec l'Islam et sa doctrine se sont faits dans le cadre de son enquête sur l'établissement des religions et de ses recherches métaphysiques commencées vers 1734 . Voltaire cherche à donner les fondements historiques et philosophiques nécessaires à son option déiste mais tout aussi bien à alimenter sa lutte pour une religion naturelle débarrassée de la superstition. Au cours du mois de juillet 1738, Turner lui envoie d'Angleterre le Koran de Sale ; l'auteur y résume dans sa préface la vie de Mahomet et commente les dogmes de la religion musulmane. Pour la première fois, Voltaire prend connaissance

plusieurs de ses lettres par l'invocation musulmane, Alab akbar etc... du muezzin dans son appel à la prière), 1617, 1618, 1619, 1624, 1630, 1631, 1633, 1638, 1640, 1642, 1648 (dans laquelle Volraire attaque Pascal, comme le patriarche du * fanatisme jansénistew), 1678. Ajoutons l'ensemble des lettres de 1745 à ses amis, au pape ou au clergé romain autour de la bénédiction papale pour le Mahomet. Il s'agit des lettres no 1916 (Voltaire mentionne a M. de Valori, son correspondant, les bénédictions qu'il a reçues du Saint-Père, lui expliquant que * Sa Sainteté a pensé comme vous sur Mahomet. C'est qu'elle n'a point été séduite par les convulsionnaires *. Et Voltaire souligne malicieusement qu'il est * fort joliment avec Sa Sainteté * si bien que par un renversement ironique, * ce serait aux dévots à me demander protection pour ce monde-ci et pour l'autre. w), 1980, 1981, 1982, 1983, 1984, 1987, 1988, 2002. 
d'une interprétation différente de la tradition légendaire et dévote sur l'Islam qui dominait jusqu'ici en France et en Europe. Toutes ces lectures sont surtout destinées à nourrir l'Essai sur les mours qui ne paraîtra qu'en 1756 et dans lequel Voltaire entend renouveler le genre historique en donnant une leçon d'élargissement, de critique et de cosmopolitisme contre Bossuet, par exemple, et son Histoire universelle qui fait l'impasse sur les grandes religions de l'Orient. Les quatorze premiers chapitres de l'Essai mettront ces sources à profit, notamment les deux chapitres sur Mahomet où Voltaire caractérise avec la plus grande rigueur et un grand souci d'objectivité le personnage et la doctrine.

Toujours est-il que ses recherches sur I'Islam et sur la personne de son fondateur donnent l'idée à Voltaire de faire "quelque chose de beau ", une tragédie qui, s'inspirant d'un épisode plus ou moins véridique de la vie de Mahomet, s'attaque «à cette espèce d'imposture qui met en ouvre à la fois l'hypocrisie des uns, et la fureur des autres $[\ldots]^{17}$. Et Voltaire, tout en faisant remarquer à Frédéric II, le roi-philosophe auquel il s'adresse, que la tragédie ne consiste pas uniquement dans une déclaration d'amour, une jalousie, et un mariage mais aussi à mettre l'borreur sur le théâtre, de se demander: «ne peut-on pas remonter, jusqu'à ces anciens scélérats fondateurs illustres de la superstition, et du fanatisme, qui les premiers ont pris le couteau sur l'autel pour faire des victimes de ceux qui refusaient d'être leurs

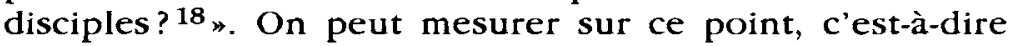
l'exploitation voltairienne des mêmes événements, documents ou témoignages, l'écart entre sa démarche critique à dominante historique et celle à dominante idéologique. Le recul en France, sous l'influence de Voltaire, de l'interprétation dévote du mahométisme et le succès de la nouvelle bistoire qu'il a ainsi introduit en pionnier, méritent toutefois d'être signalés. On le voit dès que l'on compare l'article "Alcoran" du tome I de l'Encyclopédie, paru en 1751, et dans lequel l'auteur anonyme s'appuie sur Maracci, le chef de file de la tradition dévote pour insister sur la fausseté du livre sacré des musulmans, avec l'article "mahométisme" de 1765 , qui, sous la plume de Jaucourt, s'inspire des enquêtes historiques de Voltaire pour présenter sous un jour assez favorable et généralement objectif les principales caractéristiques de la religion musulmane.

17. VOLTAIRE, Correspondance, op. cit., lettre no 1474, à Frédéric II, 20 janvier 1740 .

18. Ibidem. 
Les sources que Voltaire a utilisées 19 et qui, dans le cas de Mabomet, ressortissent à deux traditions opposées, lui fournissent le cadre ${ }^{20}$ factuel, la trame de l'action ainsi que la conception générale des principaux personnages; le travail de sélection idéologique le fera uniquement retenir les commentaires qui vont à l'appui des thèses présentant Mahomet comme un prophète imposteur dont l'ambition politique le conduit à se servir des forces de la religion pour soumettre le monde et les hommes. Dans sa pièce, les fins idéologiques l'emportent sur les moyens historiques. Ce qui importe c'est la révolution des idées et le triomphe de l'esprit philosophique sur la superstition; l'enjeu essentiel étant comme toujours d'assurer la victoire de la raison humaine contre toutes les puissances obscurantistes. Au premier rang, figurent la religion en tant qu'institution sociale et le prêtre fanatique : «on a vu dans ce même siècle où la raison élève son trône d'un côté, le plus absurde fanatisme dresser encore ses autels de l'autre ${ }^{21}$. „ Et que si on lui reproche d'avoir été emporté par le zèle, d'avoir arrangé les faits, enfin d'avoir fait commettre à Mahomet un crime dont il ne fut pas coupable, Voltaire, toujours dans sa longue lettre à Frédéric II de janvier 1740 , déjà citée, se rebiffe pour s'en expliquer ainsi : «Je sais que Mahomet $n$ 'a pas tramé précisément l'espèce de trahison qui fait le sujet de cette tragédie, l'histoire dit seulement qu'il enleva la femme de Séide, l'un de ses disciples, et qu'il persécuta

19. Voltaire a eu affaire aux représentants de deux traditions opposées : outre le livre de Sale deja mentionné, on retrouve, d'un coté, Boulainviller très favorable au Prophète et qui voit en lui la réponse à une attente messianique du peuple arabe; de l'autre, Gagnier qui défend l'interprétation traditionnelle et la thèse de l'imposture. Voltaire trouve chez ce dernier l'histoire de Zaïd qui deviendra Séide. Soit dit en passant, le nom de Séide est devenu depuis la tragédie de Voltaire et son impact sur les esprits, un nom commun pour désigner une personne au dévouement aveugle et fanatique [Voir le Dictionnaire étymologique de la langue française, PUF, 1960]. De Boulainviller, Voltaire ne retient pour la conception générale de son personnage qu'un point mais relativement important car il confere à son Mahomet une dimension politique d'une certaine grandeur. C'est la scène 5 de l'acte II dans laquelle Mahomet pour séduire Zopire qui n'est pas dupe de son role de prophète, lui révèle ses ambitieux desseins. La décomposition de l'empire romain, la faiblesse de l'Inde, de la Perse et de l'Égypte, ouvrent la voie à la grandeur arabe. Patriote, Mahomet veut lancer son peuple à la conquete du monde. Il connaît les hommes. Il sait que les fanatiques sont invincibles. Il s'est donc fait le prophète d'une religion nouvelle qui inspire l'héroïsme et dévoue le soldat, corps et ame, à son chef. Pour toute la question des sources er de leur utilisation par Voltaire dans Mahomet, voir R. Pomeau, La religion de Voltaire, nouvelle édition revue et mise a jour, Nizet, Paris, 1974, pp. 149-151 ainsi que la lettre de Voltaice à Frédéric II du 20 janvier 1740, in Correspondance, op. cit., no 1474.

20. VOLTAIRE, Correspondance, op. cit., lettre no 1327 à d'Argenson, 26 janvier 1740 , a Bruxelles.

21. VOLTAIRE, Correspondance, op. cit., lettre no 1474, à Frédéric II, roi de Prusse, 20 janvier 1740 
Abusoftan, que je nomme Zopire. Mais quiconque fait la guerre à son pays, et ose la faire au nom de Dieu, n'est-il pas capable de tout? Je n'ai pas prétendu seulement mettre une action vraie sur la scène mais des moeurs vraies, faire penser les hommes comme ils pensent dans les circonstances où ils se trouvent, et représenter enfin ce que la fourberie peut inventer de plus atroce, et ce que le fanatisme peut exécuter de plus horrible: Mahomet n'est ici autre chose que les Tartuffe les armes à la main ${ }^{22}$."

Dès lors, et puisqu'un tel esprit l'animait en composant cet ouvrage, tout est de bonne guerre pour étouffer ces germes capables d'infecter la terre. Voltaire compose avec Mabomet une peinture atroce, se vantant d'avoir été plus loin dans l'horreur ${ }^{23}$ que sur aucun théâtre. Qu'on en juge. Il résume lui-même ainsi l'action de sa tragédie: "C'est un jeune homme né avec de la vertu [Séide enlevé à son père Zopire lorsqu'il était enfant vénère en Mahomet son bienfaiteur et l'envoyé de Dieu], qui séduit par son fanatisme, assassine un vieillard qui l'aime, et qui dans l'idée de servir Dieu se rend coupable sans le savoir d'un parricide; c'est un imposteur [Mahomet présenté comme jouant le rôle de prophète et qui détourne la force de la religion au profit de ses ambitions personnelles, pour mieux asservir les hommes] qui ordonne ce meurtre, et qui promet à l'assassin un inceste pour récompense ${ }^{24}$. " Il s'agit de Palmire, fille de Zopire sans le savoir, et qui est retenue prisonnière à la Mecque; Mahomet l'aime et pense que le moyen le plus rapide de s'en emparer est de faire assassiner Zopire par Séide, et ensuite d'empoisonner son rival pour s'en débarrasser. Cette dernière forfaiture va tourner à son avantage. En effet, Séide qui a été détrompé par Zopire mourant, se retourne contre Mahomet et excite le peuple contre ce dernier. Mais au moment même où tout va mal pour Mahomet, celui-ci aperçoit Séide qui chancelle sous les effets du poison aux lents effets qu'on lui a administré à son insu. Mahomet en appelle aussitôt au jugement de Dieu : *De

22. Ibidem.

23. Ce qui choquera Jean-Jacques Rousseau plus sensible sans doute au rôle édifiant de la littérature, et à celui du théatre en particulier, qu'aux valeurs plus purement esthétiques. Voir La lettre à M. d'Alembert sur son article Genève Rousseau craint, dit-il, à propos du Mabomet de Voltaire aqu'aux yeux des spectateurs sa [celle de Mahomet] grandeur d'ame ne diminue beaucoup l'atrocité de ses crimes; et qu'une pareille pièce, jouée devant des gens en état de choisir, ne fit plus de Mahomet que de Zopire. Ce qu'il y a, du moins, de bien sür, c'est que de pareils exemples ne sont guère encourageables pour la vertu..

24. VOLTAIRE, Correspondance, op. cit., lettre no 1474, à Frédéric II, roi de Prusse, 20 janvier 1740 . 
nous deux, à l'instant que le coupable expire ${ }^{25}$. Séide s'écroule, et Mahomet utilise ce faux miracle pour annoncer à la foule qui s'apaise que Dieu lui-mème l'a déclaré son prophète et puni son adversaire. Voltaire insiste avec les dernières paroles de Mahomet sur la forfaiture essentielle des Tartuffe qui enchaînent les hommes au moyen des fers de la religion :

* Dieu, que j'ai fait servir au malheur des humains Adorable instrument de mes affreux desseins...

J'ai trompé les mortels, et ne puis me tromper...

Je dois régir en dieu l'univers prévenu

Mon empire est détruit, si l'homme est reconnu 26

Le personnage hypocrite que dépeint Voltaire ressemble plus à première vue à celui représenté par la tradition dévote. Les prélats de Lille avaient raison de se sentir en pays de connaissance. Voltaire, pourtant, a mis habilement en place les moyens d'une seconde lecture : tout en se servant de l'interprétation des dévots, il la retourne contre eux. Pourquoi l'explication du triomphe de la religion musulmane par la puissance de l'imposture ne vaudrait-elle pas pour celle de Jésus-Christ? Voltaire commence en déterminant le terrain commun aux défenseurs et aux adversaires chrétiens du mahométisme, celui de la puissance de l'imposture; ensuite il suggère, par quelques vers équivoques semés ici et là dans sa tragédie, qu'on peut ajouter à la liste des imposteurs, Jésus-Christ et les apôtres. Mahomet, par exemple, se présente comme l'envoyé de Dieu promis par les oracles : "Tu connais quel oracle et quel bruit populaire ont promis l'univers à l'envoyé d'un Dieu 27 \%. Il fait son entrée dans la Mecque, "le front calme et serein", "et l'olive à la main ${ }^{28}$ *. Ses disciples errent "de cités en déserts»; "proscrits, persécutés, bannis, chargés de fers", ils " promènent] leur fureur, qu'ils appellent divine ${ }^{29}$. Voltaire joue sur les deux registres à la fois et tout en se faisant entendre des "philosophes", il se fait applaudir des dévots, un peu naifs. On en trouvera un témoignage dans une lettre très intéressante de Lord Chesterfield, un libertin anglais, à Crébillon; la lettre est datée du 26 août 1742 et fait allusion à des faits remontant à juin 1741 environ. Les remarques de ce témoin éclairé et loin d'être dévot, mais moins radical ou

25. VOlTAIRE, Mabomet, acte V, scène 4, in Theâtre, Firmin Didot, Paris, 1853, p. 345

26 Ibidem, p. 347

27. Ibidem, acte IV, scène 3

28. Ibidem, acte II, scène 3 .

29. Ibidem, acte I, scène 4 . 
plutôt moins passionné que Voltaire donnent bien le ton de la réception de Mabomet par les habitués des "salons" parisiens, encore réticents à aller si loin contre la religion et les mœurs de leurs pères :

«Voltaire m'a récité l'année passée à Bruxelles plusieurs tírades de son Mahomet, où j'ai trouvé de très beaux vers, et quelques pensées plus brillantes que justes; mais j'ai d'abord vu qu'il en voulait à Jésus-Christ, sous le caractère de Mahomet, et j'étais surpris qu'on ne s'en fût pas aperçu à Lille, où elle fut représentée immédiatement avant que j'y passasse. Même je trouvai à Lille un bon catholique, dont le zèle surpassait la pénétration, qui était extrêmement édifié de la manière dont cet imposteur et ennemi du christianisme était dépeint [...] mais ce que je ne lui pardonne pas, et qui n'est pas pardonnable, c'est tous les mouvements qu'il se donne pour la propagation d'une doctrine aussi pernicieuse à la société civile que contraire à la religion générale de tous les pays. Je doute fort s'il est permis à un homme d'écrire contre le culte et la croyance de son pays, quand même il serait de bonne foi persuadé qu'il y eût des erreurs, à cause du trouble et du désordre qu'il y pourrait causer; mais je suis bien sûr qu'il n'est nullement permis d'attaquer les fondements de la morale, et de rompre des liens si nécessaires, et déjà trop faibles pour retenir les hommes dans le devoir ${ }^{30}$."

Les adversaires de Voltaire, plus puissants et plus perspicaces que ce bon père jésuite qui courait partout dans la ville en soutenant que le nom de Mahomet contenait le même nombre de syllabes que celui de Jésus-Christ, parviendront, à la suite d'interventions pressantes et répétées auprès du cardinal de Fleury, à faire interdire Mabomet. C'est le parti janséniste qui surtout se démène, s'étant aisément reconnu à travers les nombreux traits anti-jansénistes de la tragédie. Le Dieu vengeur et redoutable de Mahomet ressemblait fort, en effet, à celui de Jansénius : "Qu'on adore mon Dieu, dit Mahomet à ses officiers, mais surtout qu'on le craigne ${ }^{31}$.» Quant au faux miracle grâce auquel Mahomet retourne la situation en sa faveur en se prétendant par là l'élu véritable de Dieu, toute personne un peu avertie reconnaissait là les miraculés de Saint Médard et ses lamentables convulsionnaires. De même, dès la première scène de sa

30. Cité par Besterman en note 3 de la lettre de Voltaire à d'Argental, no 1538, 5 juin 1741 , et dans laquelle Voltaire rassure d'Argental qui manifestait des inquiérudes au sujet de la façon dont, avec Mabomet, Voltaire sapait en meme temps que les bases de la religion, les bases de la morale, gardiennes toutes deux de l'ordre social. In Voltaire, Correspondance, op. cit., pp. 1466-67. Besterman ajoute comme suit, à la fin de sa note, la référence de la lettre de Chesterfield : Chesterfield, Miscellanous works, Londres, 1777, t. II, p. 35-36.

31. VOLTAIRE, Mabomet, acte II, scène 3, in Theatre, op. cit. 
tragédie, Voltaire faisait de transparentes allusions aux miracles et aux prodiges que faisent les jansénistes sur la tombe de Saint Pâris, au début du siècle. Phanor annonçant à Zopire que Mahomet est prêt d'arriver à la Mecque, lui prêche ainsi la soumission devant l'irrésistible avance de son adversaire :

"Imposteur à la Mecque, et prophète à Médine,

Il sait faire adorer à trente nations

Tous ces mêmes forfaits qu'ici nous détestons.

Que dis-je? en ces murs mêmes qu'une troupe égarée

Des poisons de l'erreur avec zèle enivrée,

De ces miracles faux soutient l'illusion,

Répand le fanatisme et la sédition,

Appelle son armée, et croit qu'un dieu terrible

L'inspire, le conduit, et le rend invincible ${ }^{32}$..

Dévots et jansénistes dénoncent alors sans se tromper précisément ce que Voltaire avait comme dessein dans sa pièce. Les conséquences sociales et politiques que Chesterfield apercevaient dans cette attaque de la religion institutionnelle comme un ensemble de tromperies avait bien pour but de ruiner du même coup l'autorité de ses chefs terrestres et la crédibilité de leur enseignement. Voltaire dont le déisme ne fait pas de doute rencontre ici à l'avance l'anticléricalisme farouche de d'Holbach et de son cercle d'athées pour lesquels le chrétien superstitieux est l'antithèse du philosophe que seul la raison fait agir. Et quant à la vertu, la loi naturelle ou les principes moraux, il suffit, pour le parti de l'humanité, de respecter les commandements de la nature et d'en honorer les lois. Mais pour les tenants de l'ordre établi, Voltaire qui s'est effectivement arrangé pour que Mabomet puisse insensiblement "conclure de la religion musulmane à la nôtre», et, par là, qui menace d'ébranler les piliers de la société, doit être aussitôt réduit au silence. Le procureur général Joly de Fleury, affilié au parti janséniste, s'empresse de faire un rapport. Au reste, ce sera selon la même ligne d'argumentation que près de vingt ans plus tard, le 23 janvier 1759 exactement, le même personnage demandera au Parlement la condamnation de l'Encyclopédie, au nom de la défense de la Société toute entière puisque tout ce qui menace la Religion menace l'État et réciproquement ${ }^{33}$. "C'est l'énormité en fait d'infamie, de

\section{Ibidem, acte I, scène 1 .}

33. Joly de Fleury dit notamment dans son réquisitoire: "La Société, l'État et la Religion se présentent aujourd hui au Tribunal de la Justice pour lui porter leurs plaintes [...] Religion et fanatisme sont des termes synonymes, et le christianisme n'inspire qu'une fureur insensée qui travaille à detruire les fondements de la société.» 
scélératesse, d'irréligion et d'impiété ; et c'est ce que disent même ceux qui n'ont pas de religion ${ }^{34^{*}}$, s'indigne Joly de Fleury. Dans les notes en marge de son réquisitoire contre Mabomet, relevant ces vers de Voltaire :

«Ne donnons point le temps aux mortels détrompés

De rassurer leurs yeux de tant d'éclats frappés ${ }^{35}$.»

il s'exclame: «Qu'y a-t-il de plus énorme contre la religion?». Il poursuit en faisant grief à Voltaire d'avoir mis sur la scène publique des crimes qui font horreur à l'humanité, et d'avoir présenté son «héros" comme le "plus habile scélérat», tandis que ses victoires peuvent encourager les spectateurs à le suivre: "Quand on ajoute la connaissance que tout l'univers a des progrès de la religion mahométane, fondée par un tel scélérat, devait-on jamais montrer au public les crimes de ce malheureux, dont les suites ont été telles qu'on les connaît? ${ }^{36}$ "

Mabomet appartient à la période de Cirey, c'est-à-dire à la période pendant laquelle Voltaire confiant dans la proche généralisation du déisme parmi les couches avancées de la société, n'est pas encore entré en guerre ouverte contre l'Infâme. Il mise sur l'évolution rapide d'un christianisme de tendance plus rationnelle et plus épurée en accord avec les progrès d'une philosophie si humaine [la sienne et celle que les Encyclopédistes mettront de l'avant] pour adoucir et perfectionner les mœurs. Aussi le poète, doublé du Philosophe, dirige surtout ses coups, depuis l'ode Sur la superstition, contre ce qu'il a, et aura toujours en horreur, le fanatisme religieux; comme dans Zaïre, sa tragédie turcochrétienne, comme dans Mabomet, mais aussi comme dans La Henriade. Plutôt que la religion musulmane ${ }^{37}$, ou même la religion chrétienne, Voltaire vise une cible autrement importante à ses yeux: "l'enfant dénaturé» de toute religion, qui, "armé pour la défendre, cherche à la détruire, // Et reçu dans son sein, l'embrasse et la déchire».

34. Voir P. Bondois qui a publié le brouillon du rapport de Joly de Fleury : * Le procureur général Joly de Fleury et le Mabomet de Voltaire, in Revue d'bistoire littéraire de la France, t. XXXVI, 1929, p. 249

35. VOLTAIRE, Mahomet, acte II, scène 4, in Theatre, op. cit.

36. BONDOIS, P. op. cit., p. 248, p. 257.

37. Voir, à cet égard, l'étude très informée de Djavad Hadidi, Voltaire et l'Islam, Publications orientalistes de France, 1974, avec une préface par Étiemble. C'est un ouvrage qui Eclaire fort bien les relations de Voltaire et de l'Islam et qui les mettant en perspective avec les positions du philosophe à l'égard du christianisme, retrace l'influence de l'islamisme sur les idées religieuses de Voltaire et leur évolution au fur et à mesure que s'élargissait sa connaissance du monde musulman. 
Mettant en rapport superstition et fanatisme, Voltaire, dans l'article *fanatisme* de son Dictionnaire philosophi$q u e$, détermine le fanatisme comme la mise en action sanglante de la superstition ${ }^{38}$. Il écrit : «le fanatisme est à la superstition ce que le transport est à la fièvre, ce que la rage est à la colère [...] celui qui soutient sa folie par le meurtre est un fanatique. 39 , De même, la superstition est une outrance qui fait dépasser au superstitieux les bornes d'une religion naturelle et rationnelle : * la superstition, comme la définit Voltaire dans l'article superstition, est presque tout ce qui va au-delà de l'adoration d'un Être suprême et de la soumission du cœur à ses ordres éternels 40 ." C'est pourquoi il y a toujours sous le superstitieux, le fanatique qui ne demande qu'à s'éveiller : "le superstitieux est gouverné par le fanatique et le devient ${ }^{41}$." Les exemples qu'aligne Voltaire, à l'appui de son argumentation, dans les articles précités, répètent ceux qu'il donnait déjà à ses correspondants de 1742 , Frédéric II, César de Missy ${ }^{42}$ au moment de la querelle de Mahomet, c'est-à-dire de Tartuffe le Grand. L'inlassable dénonciation des prêtres sanguinaires du che-

38. VOLTAIRE, Dictionnaire philosopbique, (1764) édition René Pomeau, Paris, Garnier-Flammarion, 1964, article «fanatisme *, pp. 189-191. Deleyre, *l'auteur de l'analyse de la philosophie du chancelier Bacon -, avait développé certe même mise en rapport dans son très long article de l'Encyclopédie, tome VI, 1756 . Il définit le fanatisme, la bete noire du siècle des Pbilosopbes, comme un * zèle aveugle et passionne, qui nait des opinions superstitieuses et fait commertre des actions ridicules, injustes et cruelles, non seulement sans honte et sans remords mais encore avec une sorte de joie et de consolation.. Deleyre fait ensuite l'histoire du fanatisme et de ses funestes exploits, évoquant ici à la suite du Mabomet de Voltaire, et presque dans les memes termes, l'exemple du fanatisme qui l'Alcoran d'une main et le glaive de l'autre, marche a la conquête de l'Asie et de l'Afrique. - Il peut êre intéressant pour éclairer ce qui pour les contemporains apparaissait comme le centre de gravité de la pièce de Volraire, de faire aussi remarquer que pour Deleyre, comme pour Voltaire, Mahomet était *d'abord un fanatique, et puis un imposteur.. L'article de l'Encyclopédie évoque ensuite de nombreux autres exemples de fanatisme religieux pris dans l'histoire occidentale, notamment les horreurs de la * nuit de la Saint-Barthélémy *; pour les deux auteurs des articles en cause, les excès de cette nuit funeste demeurent * le plus détestable exemple de fanatisme *. Deleyre definit ensuite le concept et détermine les *sources* du fanatisme. Il les trouve principalement dans la religion et l'intolérance pour conclure en affirmant que "la vérité ne fait point de fanatiques [...] esprit outré, le fanatisme a fait beaucoup plus de mal au monde que l'impiete, ennemi du genre humain *. De meme Voltaire conclura son article *superstition * par cette declaration : "en un mot, moins de superstitions, moins de fanatisme; et moins de fanatisme, moins de malheurs. In Dictionnaire pbilosopbique, op. cit., p. 360

39. VOLTAIRE, Dictionnaire pbilosopbique, (1764), op. cit., article * fanatisme*, p. 189.

40. VOLTAIRE, Dictionnaire pbilosopbique, (1764), op. cit., article * superstition *, p. 357

41. Ibidem, p. 358.

42. VOLTAIRE, Correspondance, op. cit., lettre no 1617, à Frédéric II, roi de Prusse, 29 aoùt 1742; lettre no 1618 , à Cesar de Missy, 1 septembre 1742 , lettre no 1619 , a Cideville, 1 septembre 1742 . 
valier de La Barre et de Calas, trouve en Mahomet sa frappante métaphore; et Voltaire représente avec férocité dans sa tragédie cet autre "prieur des Jacobins " 43 qui nourrit de fanatisme le jeune courage de Séide pour en faire l'instrument et la victime du crime :

Du plus saint appareil la ténébreuse horreur

Les autels, les serments, tout enchaîne Séide

J'ai mis un fer sacré dans sa main parricide,

Et la religion le remplit de fureur ${ }^{44}$.

Ici, comme dans La Henriade, c'est René Pomeau qui le fait justement remarquer, "Voltaire est inspiré par son horreur de la passion religieuse 45 ». Il a recours aux mêmes images de sanctuaires ténébreux et sanglants, il convoque les mêmes visages haineux de fanatiques, moines fourbes, peuple déchaîné, grands exaltés, qui viennent se cristalliser dans les personnages représentés dans Mabomet. Voltaire a en abomination tous ces perfides qui, pareils à Mahomet, et il suffit de transporter le nom du Livre sacré dont ils savent couvrir leurs crimes, «le glaive et l'Alcoran dans [leurs] sanglantes mains // imposent silence au reste des hu-

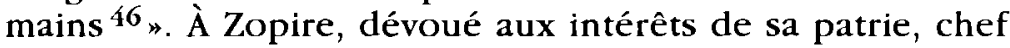
dévoué de gouvernement, qui joue le même rôle que le valeureux Henri III, faisant la guerre contre la Ligue, s'oppose Mahomet, le monstre aux complots perfides. Voltaire peint le duc de Guise sous les mêmes sombres couleurs. Comme Mahomet, le duc de Guise était le chef d'une troupe

43. VOLTAIRE, Correspondance, op. cit., lettre no 1618, à César de Missy, 1 septembre 1742 : * J'ai voulu faire voir par cet ouvrage [Mabomet] à quels horribles excès le fanatisme peut entraîner des ames faibles conduites par un fourbe; ma pièce représente sous le nom de Mahomet le prieur des Jacobins mettant le poignard à la main de Jacques Clément, encouragé de plus par sa maîtresse au parricide. On reconnaît là, l'auteur de La Henriade, mais il faut que l'auteur de La Henriade soit persécuté, car il aime la vérité et le genre humain. Il n'est permis aux poètes d'être philosophes qu'à Londres. - On rapprochera cette lettre de celle que 15 ans plus tard, Voltaire écrira à d'Alembert, avocat comme lui du déisme rationnel et d'une religion naturelle purgée de toute violence. $\grave{A}$ la suite du scandale soulevé par l'article * Genève * (tome VII de l'Encyclopédie, 1757) de l'Alembert, Voltaire s'emporte contre les pasteurs protestants et leur esprit d'intolérance, son anticléricalisme, ses fantasmes des pretres sanguinaires qui excitent le fanatisme populaire deja transposés depuis La Henriade et Mabomet, apparaissant crument dans sa lettre au sujet de la nouvelle cabale "Ces gens-là vont se couvrir de ridicule; chaque démarche qu'ils font depuis le tombeau du diacre Paris, la place où ils ont assassiné Servet, et jusqu'à celle où ils ont assassiné Jean Hus, les rend tous également l'opprobre du genre humain. Fanatiques papistes, fanatiques calvinistes, tous sont petris de la meme $\mathrm{m}$... détrempee de sang corrompu. . In Correspondance, op. cit., tome IV, 1978, lettre no 4965, à d'Alembert, 12 décembre 1757.

44. VOLTAIRE, Mabomet, acte III, scène 5, in Theâtre, op. cit.

45. POMEAU, R., La religion de Voltaire, op. cit., p. 153.

46. VOLTAIRE, Mabomet, acte II, scène 5, in T7éátre, op. cit. 
de fanatiques ; "trop habile et trop savant à nuire", il n'hésite pas, tout comme Mahomet, en apportant le carnage et l'effroi, à commander aux bumains de penser comme lu ${ }^{47}$. Les concordances avec La Henriade sont nombreuses et délibérées. Elles culminent dans la figure de Séide, choisi parmi les autres jeunes musulmans pour son aveuglement et sa docilité les plus grands, pour assassiner Zopire. «De ses décrets divins, ordonne Mahomet à Séide le poussant au parricide, aveugle exécuteur, // Adorez et frappez.» Aussitôt Séide de répondre :

"Je crois entendre Dieu ; tu parles, j'obéis ${ }^{48}$."

Les modèles occidentaux de Séide, et Voltaire ne se fait pas faute de les rappeler à ses interlocuteurs pendant que fait rage la bataille de Mabomet, reprennent les personnages de La Henriade. La psychologie de Séide est presque identique à celle de Jacques Clément, ou à celle de Ravaillac tels que les évoque Voltaire; ces jeunes gens abusés par leurs maîtres sont persuadés tout comme Séide séduit par Mahomet, du caractère sacré de leur mission et courent en aveugle tuer le roi qui ne pense pas comme leurs maîtres.

C'est qu'il s'agit dans Mabomet comme dans La Henriade de ce qui est, et demeurera le grand combat politique de toute la vie de Voltaire, celui de la raison "contre les puissances obscures». Les maximes que le philosophe met dans la bouche de Mahomet, construisent le prototype du prêtre criminel qui exige des fidèles une soumission aveugle et qui, au nom de Dieu, les exalte à tuer :

" $[\ldots]$ Téméraire,

On devient sacrilège alors qu'on délibère.

Loin de moi les mortels assez audacieux

Pour juger par eux-mêmes, et pour voir par leurs yeux!

Quiconque ose penser n'est pas né pour me croire.

Obéir en silence est votre seule gloire ${ }^{49}$."

Dans la France du XVIII siècle, au temps du jansénisme convulsionnaire, des persécutions contre les protestants, du supplice de Calas, roué, étranglé et brûlé, de La Barre décapité et sur le corps duquel on brûlera le Dictionnaire Pbilosophique, Voltaire s'attaque à des manifestations bien réelles de la * folie religieuse * et d'un fanatisme populaire qui renouvelle les horreurs de la Ligue. Et à Voltaire-Zopire adjurant Omar qui est venu pour exiger sa soumission à 
Mahomet: "Sers-toi de ta raison, juge avec moi ton maître ${ }^{50}$, fera sans cesse écho l'exhortation de VoltaireBoldmind : "Osez penser par vous-mêmes. Nous ne sommes heureux en Angleterre que depuis que chacun jouit librement du droit de dire son avis." Aussi bien les articles «fanatisme» ou «superstition" du Dictionnaire ne voyaient comme remède aux fureurs du fanatisme, à *cette maladie épidémique, que l'esprit philosophique, qui, répandu de proche en proche, adoucit enfin les mours des hommes, et qui prévient les accès du mal ${ }^{51}$. $E t$, contre les excès de la religion, le recours à une religion épurée et rationnelle, telle celle "des lettrés de la Chine" qui donne le seul exemple dans le monde d'«une religion qui n'ait pas été souillée par le fanatisme 52 .» Le triomphe de la lumière naturelle dans le conflit qui l'oppose au fanatisme s'associe ainsi à l'idée d'un Dieu clément, aux bienfaits qui procèdent de la tolérance et de l'amour entre frères humains. L'horreur du fanatisme, la confiance de Voltaire dans les garanties que procure la loi naturelle conçue comme le fonds commun de l'humanité, la liberté absolue de penser pour laquelle il se bat, tou te son action se tend vers l'établissement de cette cité d'hommes, libres enfin de tous préjugés superstitieux, de cette cité du bonheur pour laquelle luttent les Philosophes, et au sein de laquelle, sous le sûr rempart des lois, vivrait, prospère et tranquille, tout le genre humain.

\section{"L'amour du genre humain et l'horreur du fanatisme..."}

«Ceux qui diront que les temps de ces crimes sont passés, déclarait Voltaire à Frédéric II, qu'on ne verra plus de Barcochebas, de Mahomet, de Jean de Leyde, que les flammes des guerres de religion sont éteintes, font ce me semble trop d'honneur à la nature humaine. Le même poison subsiste encore quoique moins développé. Cette peste qui semble étouffée reproduit de temps en temps des germes capables d'infecter la terre. N'a-t-on pas vu de nos jours les prophètes des Cévennes tuer au nom de Dieu ceux de leur secte qui n'étaient pas assez soumis ?53"

50. Ibidem, acte I, scène 4.

51. VOLTAIRE, Dictionnaire pbilosopbique, (1764), op. cit., article "fanatisme *, p. 190 .

52. Ibidem.

53. VOLTAIRE, Correspondance, op. cit., lettre no 1474 à Frédéric II, roi de Prusse, 20 janvier 1740 . 
$\mathrm{Au} \mathrm{XX} \mathrm{X}^{\mathrm{e}}$ siècle ce sont les hommes politiques, récemment doublés d'hommes religieux, qui fanatisent les masses et s'emparent de leur volonté. Les tristes crimes dont ne sont exempts aucun de nos pays modernes ont malheureusement rendu de l'actualité aux remarques de Voltaire. Mais le procès qu'il a fait sa vie durant aux forces mauvaises qui enchainent les hommes et font leur malheur, son nom qu'il a lié au concept même de tolérance - tolérance de combat dans le Dictionnaire comme dans Mabomet, et, de ce fait, assez peu tolérante parfois ${ }^{54}$-, les valeurs, les droits, les devoirs, inhérents à l'être même de l'homme, qu'il a prônés sans relâche, font accéder les idées de Voltaire, plutôt qu'à une actualité transitoire, à une véritable éternité.

Le Philosophe par excellence espérait se voir récompensé si son ouvrage pouvait encourager les âmes à se rebeller devant la malheureuse obéissance de Séide, et à se dire : "pourquoi obéirais-je en aveugle à des hommes aveugles qui me crient haissez, persécutez, perdez celui qui est assez téméraire pour n'être pas de votre avis et même sur des choses indifférentes que nous n'entendons pas 55 ". Affirmons à notre tour que chaque fois que cet "esprit d'indulgence qui fait des frères * prévaut sur celui "d'intolérance qui forme des monstres $»$, la voix de Voltaire ne s'est pas faite entendre en vain. Son cuvre milite, avec allégresse, avec efficacité, voilà bientôt trois siècles aujourd'hui, contre le fanatisme, les injustices et les cruautés. Et comme il le souhaitait en adressant son Mabomet à Frédéric II, à ce roi dont le gouvernement exemplaire aux yeux du siècle se réclamait de la force de la raison, la pièce de Voltaire, manifeste et symbole tout à la fois, dictée par l'horreur des Lumières envers le fanatisme et la superstition, aura «servi à déraciner de tels sentiments chez les hommes ${ }^{56}$.

Département de philosopbie

Université du Québec à Montréal

54. Comme on le sait, *il est exceptionnel qu'un principe n'ait pas à patir des luttes qui tendent à l'imposer $*$, et celui de tolérance n'échappe pas à la règle. Claudel le laissait vertement entendre qui disait que pour la tolérance il y auait des maisons...!

55. Ibidem.

56. Ibidem 